\title{
Características sensoriais e efeito da informação do conteúdo de gordura sobre a aceitação sensorial em salsichas tipo Frankfurt
}

\section{Sensorial description and effect of the light label on the sensorial acceptability of Frankfurt sausage}

\author{
Isabela Ciarlini de Azevedo, ${ }^{*}$ Mônica Queiroz de Freitas, ${ }^{* \star}$ Sérgio Borges Mano, ${ }^{* *}$ Henrique Silva Pardi**
}

\begin{abstract}
Resumo
O presente trabalho teve como tema central a descrição sensorial de salsichas tipo Frankfurt tradicional e de carne de aves obtidas no mercado brasileiro. Dentre as salsichas de carne de aves, contendo baixo teor de gordura, estudou-se também o efeito da rotulação light sobre a aceitação sensorial de consumidores. Utilizou-se o método de análise descritiva quantitativa para a descrição sensorial das salsichas tradicionais e de carne de aves. Os testes de consumidor foram realizados em salsichas de carne de aves, com e sem a informação que se tratava de produto light, cuja aceitação foi medida em escala hedônica e de atitude. Os dados foram analisados estatisticamente pela análise de variância e pela Análise de Componente Principal (ACP). No geral, as salsichas tradicionais apresentaram características de sabor e aroma mais acentuados que as produzidas com carne de aves. Os atributos granulosidade, sabor de soja e sabor de ranço foram considerados negativos para a qualidade sensorial das salsichas estudadas. Os resultados da ACP sugerem que os atributos: aroma característico, sabor característico e cor rósea foram os mais importantes na diferenciação das salsichas tradicionais das salsichas produzidas com carne de aves. A informação nutricional quanto ao conteúdo de gordura ou light demonstrou uma interferência levemente negativa no status afetivo das salsichas produzidas com carne de aves $(p<0,05)$, não tendo sido observada modificação significativa $(p>0,05)$ na atitude de freqüência de uso dos consumidores testados. Um grupo maior de consumidores (87\%) tendeu à aceitação para as amostras de chester e de frango, e apenas 13\% para as amostras de peru.
\end{abstract}

Palavras-chave: salsicha, análise sensorial, análise descritiva, aceitação, intenção de compra, baixo teor de gordura.

\begin{abstract}
This present research had as his central theme the sensorial description of conventional and low-fat frankfurt sausage obtained in the Brazilian marketplace. Among the low-fat sausages (produced with poultry meat), it was observed the effect of the "light" label on the sensorial acceptability of the consumer. The method for the quantitative descriptive analysis was used for the sensorial description of the conventional and low-fat sausage. The consumer's tests were made for low-fat sausages, with and without the "light" information, which acceptance was measured in hedonic scale and from purchase intent. The data were statistically analyzed by the variance analysis and the Principal Component Analysis (PCA). In general, the conventional sausages presented taste and smell characteristics more accentuated than the ones produced from poultry meat (low-fat). The granular attribute, soybean taste and rancidity taste were considered negative for the sensorial quality of the studied sausages. The PCA results suggest that the attributes: characteristic smell, characteristic taste and rose-colored color were the most important in the differentiation between the traditional sausages and the ones produced with poultry meat. The nutritional information as to the fat content or "light" label had a slightly negative interference in the affective status of the sausages produced with poultry meat $(p<0.05)$, without any observation of significant modification $(p>0.05)$ on the purchase intent of the tested consumers. A larger group of consumers (87\%) leaned to the acceptance of the Chester and chicken samples, and just $13 \%$ to the turkey samples.
\end{abstract}

Keywords: sausage, sensory analysis, descriptive analysis, acceptability, purchase intent, low-fat.

\section{Introdução}

As indústrias produtoras de alimentos preocupam-se cada vez mais em atender às expectativas do consumidor, buscando desenvolver produtos diferenciados para cada segmento do mercado. Acompanhando uma tendência mundial, uma crescente fatia do mercado nacional vem optando pelo consumo de derivados cárneos menos calóricos. Na salsicha tipo Frankfurt tradicional a gordura animal representa 20 a $25 \%$ da composição do produto, quando produzida com carne

\footnotetext{
* Programa de Pós-Graduação em Medicina Veterinária (Mestrado) - Higiene Veterinária e Processamento Tecnológico de Produtos de Origem Animal - Faculdade de Veterinária - Universidade Federal Fluminense.

** Departamento de Tecnologia dos Alimentos da Faculdade de Veterinária da Universidade Federal Fluminense. Rua Vital Brazil Filho, 64. Niterói - RJ, CEP 24230-340.

Autor para correspondência: Mônica Queiroz de Freitas. E-mail: mqueiroz@vm.uff.br.
} 
de ave o rótulo indica valores inferiores em 55 a 60\%, ou seja, com 11 a $12 \%$ de gordura. Sob o ponto de vista nutricional essa mudança é bastante desejável, porém acarreta mudanças sensoriais conforme demonstrado por Marquez et al. (1989), Barbut e Mittal (1996) e Ordóñez et al. (2001).

Portanto, os objetivos do trabalho foram: apresentar os atributos sensoriais de aroma, sabor, textura e aparência empregados na descrição de salsichas tipo Frankfurt tradicionais e produzidas com carne de aves; conhecer os atributos sensoriais importantes na diferenciação das amostras testadas; demonstrar como os consumidores se segmentam em função da aceitação por salsichas de carne de aves comercializadas no mercado nacional e; qual o efeito da rotulação light ou "baixo teor de gordura" sobre a aceitação dos consumidores.

\section{Material e métodos}

Foram escolhidas seis amostras de salsichas tipo Frankfurt comercializadas no mercado nacional, sendo duas tradicionais e quatro com baixo teor de gordura (rotulação light) de carne de frango, chester e peru, pertencentes a duas marcas distintas. As análises sensoriais foram realizadas no Laboratório de Análise Sensorial de Alimentos de Bebidas da Faculdade de Veterinária da Universidade Federal Fluminense.

Para a formação da equipe treinada de degustadores para a Análise Descritiva Quantitativa (ADQ), foi distribuída uma ficha de recrutamento em que se observou a capacidade de descrição de características sensoriais de alimentos e de uso de escalas, além da disponibilidade de tempo. A etapa de seleção dos degustadores foi realizada a partir de teste de diferença sensorial (método triangular), sob condições laboratoriais. Foram realizadas quatro repetições do teste por degustador, em que 14 degustadores, que obtiveram o mínimo de $75 \%$ de acerto no teste triangular, foram selecionados (Stone e Sidel, 1993). Na etapa de treinamento foi apresentada uma lista prévia de atributos sensoriais descritos para derivados cárneos e, a partir do método rede (Moskowitz, 1983), foram levantados estes atributos. A partir de discussões em grupo, foram definidos os atributos e a lista contendo o significado do vocabulário descritivo empregado para as amostras. Também foi produzida a ficha de avaliação com 16 atributos sensoriais dispostos em escala não estruturada de $15 \mathrm{~cm}$, ancoradas por termos de intensidade (Stone e Sidel, 1993). Nove degustadores, selecionados e treinados, foram testados quanto ao desempenho, utilizando três amostras e a ficha de avaliação produzida na etapa de treinamento (Huerta-Leidenz et al., 1996). Foi realizada a análise de variância dos dados segundo Damásio e Costell (1991). Finalmente, foram selecionados cinco degustadores, número mínimo sugerido por Moskowitz (1983), que realizaram a ADQ conforme recomendado por Stone e Sidel (1993), ou seja, com todas as amostras, apresentadas uma a uma, em quatro repetições por degustador.

O teste de aceitação sensorial foi realizado sob condições laboratoriais com 31 consumidores. Foram testadas cinco amostras de salsicha tipo Frankfurt rotuladas como light ou "baixo teor de gordura", produzidas por duas marcas competidoras do mercado nacional, duas de carne de chester, duas de peru e uma de frango. Dessas, quatro amostras eram idênticas as utilizadas pela equipe de degustadores treinados, sendo incluída mais uma salsicha de chester recentemente lançada no mercado. A ficha de avaliação continha uma escala hedônica e uma escala de atitude em relação ao consumo do alimento, ambas estruturadas e contendo nove pontos (Chaves e Sproesser, 1996). Cada amostra foi testada duas vezes por cada consumidor. No primeiro teste os consumidores não recebiam qualquer informação do produto na ficha de avaliação e, no segundo, era fornecida a informação de que se tratava de um produto light. As amostras foram codificadas com números de três dígitos e apresentadas de forma monádica e aleatorizada. Os testes foram distribuídos em sessões, com intervalos que impediam a fadiga das percepções sensoriais. Nos intervalos, os consumidores preenchiam um questionário que abordava questões de saúde, escolaridade, freqüência de consumo do produto, além da idade e do sexo, a fim de traçar um perfil geral dos participantes.

Os dados obtidos na $A D Q$ e no teste de aceitação foram tratados estatisticamente pela análise de variância e teste de média (Tukey), ao nível de $5 \%$ de significância, e pela Análise de Componente Principal (ACP). Todas as análises foram realizadas no programa estatístico SAS (1999).

\section{Resultados e discussão}

A equipe de cinco julgadores treinados, três mulheres e dois homens, empregou 16 atributos sensoriais de aroma, sabor, textura e aparência para descrever as salsichas estudadas, de acordo com o Quadro 1.

Os valores médios de intensidade de percepção dos atributos sensoriais, obtidos dos cinco julgadores em quatro repetições, estão dispostos na Tabela 1. Observa-se que os atributos considerados sensorialmente negativos para o produto-teste, como sabor de ranço (A8), sabor de soja (A9) e granulosidade na massa (A13), foram significativamente superiores nas amostras light peru marca 2 e light chester marca 2.

Como as escalas de intensidade variaram de forma crescente, observam-se perfis sensoriais mais acentuados nas salsichas tradicionais e mais suaves nas salsichas light peru marca 2 e light chester marca 2, principalmente nos atributos de aroma e sabor. As salsichas light peru marca 1 e light frango marca 1 apresentaram perfis sensoriais intermediários aos grupos anteriormente citados, porém, nos atributos de textura apresentaram acentuada maciez (A11) e coesividade (A12).

Dentre as salsichas tradicionais, a da marca 1 (M1) se destaca pelo aroma e sabor de defumado (A1 e A6) e a salsicha da marca 2 (M2) pelo aroma e sabor característicos (A3 e A7) e granulosidade da massa (A13). Nas salsichas de carne de peru e de chester da marca 2 (M2) não foi encontrada diferença significativa entre os perfis sensoriais ao nível de $5 \%$ de probabilidade. As salsichas de peru e de frango da marca 1 (M1) se diferenciaram entre si, principalmente, pela alta maciez (A11), coesividade (A12) e suculência (A14) observadas na salsicha de peru e pelo acentuado sabor de frango (A4), aroma de condimento (A2) e cor rósea (A15) observados na salsicha de frango. Na literatura consultada foi encontrado apenas o trabalho de Ordóñez et al. (2001) passível de com- 
Quadro 1: Vocabulário descritivo empregado na avaliação sensorial, pelo método de análise descritiva quantitativa, idealizado por cinco degustadores treinados, em salsicha tradicional e light comercializadas no mercado nacional

\begin{tabular}{|l|l|}
\hline \multicolumn{1}{|c|}{ ATRIBUTOS DE AROMA } & \multicolumn{1}{c|}{ DEFINIÇÃo } \\
\hline A1. De defumado & Aroma característico de produto cárneo defumado, aproximando-se ao paio. \\
\hline A2. De condimento & Aroma de condimento empregado tradicionalmente em salsicha tipo hot-dog. \\
\hline A3. Característico & Aroma característico de salsicha tipo hot-dog tradicional (carne suína e bovina). \\
\hline ATRIBUTOS DE SABOR & \\
\hline A4. Carne de frango & Sabor de carne de frango cozida. \\
\hline A5. Picante & Sensação de ardência (sabor apimentado) percebida com a amostra na boca. \\
\hline A6. De defumado & Sabor característico de produto cárneo defumado. \\
\hline A7. Característico & Sabor característico de salsicha tipo hot-dog tradicional (carne suína e bovina). \\
\hline A8. De ranço & Sabor de gordura oxidada, lembrando patê de fígado enlatado. \\
\hline A9. De soja & Sabor de proteína texturizada de soja. \\
\hline ATRIBUTOS DE TEXTURA & \\
\hline A10. Elasticidade & $\begin{array}{l}\text { Após a compressão parcial da amostra com os dentes incisivos ou molares, sem } \\
\text { rompê-la, observou-se o grau com que a amostra retornava a sua forma original. }\end{array}$ \\
\hline A11. Maciez da massa & $\begin{array}{l}\text { Na primeira mordida, com os incisivos ou molares, observou-se a força necessária } \\
\text { para atravessar uma rodela de salsicha com espessura padronizada em 15 mm. }\end{array}$ \\
\hline A12. Coesividade & $\begin{array}{l}\text { Durante a mastigação, observou-se o grau com que as partículas da amostra se } \\
\text { mantiveram coesas. }\end{array}$ \\
\hline A13. Granulosidade na massa & $\begin{array}{l}\text { Durante a mastigação e na mastigação plena, observou-se a quantidade de } \\
\text { partículas sólidas (lembrando fragmentos de ossos e cartilagens) presentes na } \\
\text { massa. Relaciona-se com a carne mecanicamente separada. }\end{array}$ \\
\hline A14. Suculência & Durante a mastigação da amostra, observou-se a quantidade de umidade liberada. \\
\hline ATRIBUTOS DE APARÊNCIA & \multicolumn{1}{c}{ DEFINIÇão } \\
\hline A15. Cor rósea & $\begin{array}{l}\text { Cor rósea característica de produto à base de carne, variando de pálido (mais } \\
\text { acinzentado) a corado (mais avermelhada). }\end{array}$ \\
\hline $\begin{array}{l}\text { A16. Homogeneidade visual } \\
\text { da massa }\end{array}$ & $\begin{array}{l}\text { Presença de fragmentos pontiagudos na superfície de corte transversal da salsicha. } \\
\text { Relaciona-se com a carne mecanicamente separada. }\end{array}$ \\
\hline
\end{tabular}

Tabela 1: Médias de atributos sensoriais descritivos em salsicha tradicional (Tradicion.) e light (L), da marca 1 (M1) e marca 2 (M2), pelo método de análise descritiva quantitativa

\begin{tabular}{lcccccc}
\hline \hline \multicolumn{1}{c}{ Atributo sensorial } & $\begin{array}{c}\text { Tradicion. } \\
\text { M1 }\end{array}$ & $\begin{array}{c}\text { Tradicion. } \\
\text { M2 }\end{array}$ & $\begin{array}{c}\text { L. Peru } \\
\text { M1 }\end{array}$ & $\begin{array}{c}\text { L. Peru } \\
\text { M2 }\end{array}$ & $\begin{array}{c}\text { L. Frango } \\
\text { M1 }\end{array}$ & $\begin{array}{c}\text { L. Chester } \\
\text { M2 }\end{array}$ \\
\hline A1. Aroma defumado & $11,9^{\mathrm{a}}$ & $3,9^{\mathrm{b}}$ & $1,6^{\mathrm{bc}}$ & $1,7^{\mathrm{bc}}$ & $1,8^{\mathrm{bc}}$ & $1,6^{\mathrm{c}}$ \\
\hline A2. Aroma de condimento & $3,7^{\mathrm{b}}$ & $5,7^{\mathrm{ab}}$ & $7,6^{\mathrm{a}}$ & $3,8^{\mathrm{b}}$ & $3,3^{\mathrm{b}}$ & $4,6^{\mathrm{ab}}$ \\
\hline A3. Aroma característico & $5^{\mathrm{b}}$ & $10,6^{\mathrm{a}}$ & $1,3^{\mathrm{c}}$ & $1,6^{\mathrm{c}}$ & $1,3^{\mathrm{c}}$ & $1,3^{\mathrm{c}}$ \\
\hline A4. Sabor de frango & $1,3^{\mathrm{d}}$ & $1,3^{\mathrm{d}}$ & $4,5^{\mathrm{b}}$ & $2,7^{\mathrm{cd}}$ & $13,7^{\mathrm{a}}$ & $3,6^{\mathrm{bc}}$ \\
\hline A5. Sabor picante & $4,2^{\mathrm{b}}$ & $4,9^{\mathrm{b}}$ & $9,3^{\mathrm{a}}$ & $3,2^{\mathrm{b}}$ & $3,9^{\mathrm{b}}$ & $5,2^{\mathrm{b}}$ \\
\hline A6. Sabor defumado & $11,9^{\mathrm{a}}$ & $3,9^{\mathrm{b}}$ & $1,3^{\mathrm{c}}$ & $1,3^{\mathrm{c}}$ & $1,8^{\mathrm{bc}}$ & $1,5^{\mathrm{c}}$ \\
\hline A7. Sabor característico & $5,3^{\mathrm{b}}$ & $13,3^{\mathrm{a}}$ & $1,3^{\mathrm{c}}$ & $1,3^{\mathrm{c}}$ & $1,3^{\mathrm{c}}$ & $1,4^{\mathrm{c}}$ \\
\hline A8. Sabor de ranço & $1,8^{\mathrm{b}}$ & $1,4^{\mathrm{b}}$ & $6,5^{\mathrm{a}}$ & $4,9^{\mathrm{a}}$ & $1,8^{\mathrm{b}}$ & $4,7^{\mathrm{a}}$ \\
\hline A9. Sabor de soja & $1,5^{\mathrm{c}}$ & $1,7^{\mathrm{c}}$ & $3,8^{\mathrm{b}}$ & $12,1^{\mathrm{a}}$ & $1,7^{\mathrm{c}}$ & $11,8^{\mathrm{a}}$ \\
\hline A10. Elasticidade & $5,5^{\mathrm{bc}}$ & $5,2^{\mathrm{bc}}$ & $3,1^{\mathrm{c}}$ & $11,3^{\mathrm{a}}$ & $6,3^{\mathrm{bc}}$ & $8,3^{\mathrm{ab}}$ \\
\hline A11. Maciez & $11^{\mathrm{ab}}$ & $10^{\mathrm{bc}}$ & $13,4^{\mathrm{a}}$ & $1,8^{\mathrm{d}}$ & $8^{\mathrm{c}}$ & $3,7^{\mathrm{d}}$ \\
\hline A12. Coesividade & $10,3^{\mathrm{b}}$ & $8,7^{\mathrm{b}}$ & $13,1^{\mathrm{a}}$ & $3,3^{\mathrm{c}}$ & $4,5^{\mathrm{c}}$ & $4,6^{\mathrm{c}}$ \\
\hline A13. Granulosidade & $3,6^{\mathrm{c}}$ & $5,7^{\mathrm{b}}$ & $1,6^{\mathrm{d}}$ & $11,3^{\mathrm{a}}$ & $2,7^{\mathrm{cd}}$ & $11,4^{\mathrm{a}}$ \\
\hline A14. Suculência & $11^{\mathrm{a}}$ & $8,9^{\mathrm{ab}}$ & $11^{\mathrm{a}}$ & $2,3^{\mathrm{c}}$ & $7^{\mathrm{b}}$ & $3,2^{\mathrm{c}}$ \\
\hline A15. Cor rósea & $9,9^{\mathrm{ab}}$ & $12,3^{\mathrm{a}}$ & $7,1^{\mathrm{bc}}$ & $4,4^{\mathrm{c}}$ & $11,6^{\mathrm{a}}$ & $3,9^{\mathrm{c}}$ \\
\hline A16. Homogeneidade visual & $4,8^{\mathrm{b}}$ & $2,7^{\mathrm{b}}$ & $12,3^{\mathrm{a}}$ & $3,8^{\mathrm{b}}$ & $13,3^{\mathrm{a}}$ & $4,9^{\mathrm{b}}$ \\
\hline \hline
\end{tabular}

* Pelo menos uma letra diferente na mesma linha indica diferença significativa na análise de variância ao nível de $5 \%$ de probabilidade $(p<0,05)$. paração, o qual descreve a dureza e a suculência como os atributos de textura mais importantes na diferenciação de salsichas tipo Frankfurt produzidas com baixo e alto teor de gordura. No presente trabalho, todos os atributos de textura participaram com intensidades semelhantes na diferenciação de amostras tradicionais e de carne de aves, discordando da conclusão de Ordóñez et al. (2001), que verificaram que os atributos dureza e suculência foram os mais importantes para definir e diferenciar a textura das amostras de salsicha utilizadas.

$\mathrm{Na}$ análise de componente principal sobre os resultados da ADQ, os componentes principais 1 e 2 acumularam 77\% da variação ocorrida entre as amostras. O mapa sensorial, mostrado na Figura 1 sugere que os atributos: aroma característico (A3), sabor característico (A7) e cor rósea (A15) foram os mais importantes na diferenciação das salsichas tradicionais das demais amostras light testadas. O mapa sensorial também sugere que os atributos mais importantes na diferenciação das amostras light peru marca 2 e light chester marca 2, das demais amostras, foram: sabor de soja (A9), elasticidade (A10) e granulosidade (A13). Vale destacar que os atributos sabor de soja (A9) e granulosidade (A13) foram considerados negativos para a qualidade sensorial do produtoteste. As amostras light peru marca 1 e light frango marca 1 destacaram-se dos demais grupos a partir dos atributos: aroma de condimento (A2), sabor picante (A5) e homogeneidade visual da massa (A16).

No geral, as salsichas tipo Frankfurt tradicionais apresentaram sabor e aroma mais acentuados que as salsichas tipo Frankfurt produzidas com carne de aves. A granulosi- 
dade e a homogeneidade visual da massa, características que se relacionam com a carne mecanicamente separada, além do sabor de ranço e de soja, foram considerados atributos negativos para a qualidade sensorial do produtoteste.

um declínio da aceitação após a informação "baixo teor de gordura", indicando um efeito negativo no "status" afetivo do produto em consumidores jovens.

A intenção de consumo dos consumidores (medido em escala de atitude) se aproximou do termo "comeria de vez em quando" (6), não apresentando modificação significativa ( $p>0,05)$ em função da informação do conteúdo de gordura. Tais resultados concordam com os obtidos por Helgensen et al. (1998), em consumidores jovens, e corroboram com os obtidos por Solhein e Lawless (1996) em consumidores com atitude positiva para o consumo de alimento com baixo teor de gordura.

A aceitação dos 31 consumidores, medida pelo status afetivo sem a informação do conteúdo de gordura, indicou que as amostras formuladas com carne de chester obtiveram aceitação superior (próximo a 7,3), a produzida com carne de frango aceitação intermediária $(6,3)$ e as produzidas com carne de peru aceitação inferior (próximo a 5,5), ao nível de 5\% de probabilidade. Agrupando-se as amostras em dois grupos, de acordo com as marcas, não houve diferença significativa na aceitação dos consumidores $(p>0,05)$.

Sabendo-se que a estratégia de vendas das grandes empresas se baseia em diversificar seus produtos para alcançar todas as fatias do mercado, o Mapa de Preferência Interno segmentou os 31 consumidores em três grupos definidos de acordo com as afinidades na aceitação pelas cinco salsichas light testadas. Um grupo maior de consumidores (65\%) tendeu à aceitação para as amostras Chester M2-A e Frango M1, 22\% para a amostra Chester M2-B e $13 \%$ para as amostras de Peru marca 1(M1) e 2 (M2). Tais resultados podem ser explicados pelo fato do atributo sabor de ranço, observado pela equipe treinada somente nas salsichas de peru e considerado sensorialmente negativo, ter sido percebido pelos consumidores.

Nas cinco amostras de salsicha light testadas, a informação do teor de gordura demonstrou uma A16: Homogeneidade visual da massa. CP1: Componente Principal 1 CP2: Componente Principal 2.

O teste de aceitação foi realizado com 31 indivíduos com idade variando entre 20 e 25 anos, $55 \%$ do sexo feminino, em que $75 \%$ consumiam o produto-teste algumas vezes e $25 \%$ muito freqüentemente, todos gozando de boa saúde e sem restrição alimentar. Os resultados do teste de aceitação indicaram que a informação light modificou significativamente $(p<0,05)$ o status afetivo (medido em escala hedônica) do produto. Observou-se que, após a informação do conteúdo de gordura, os consumidores declinaram levemente a aceitação sobre o produto. Sem a informação do conteúdo de gordura, o escore médio $(6,2)$ variou entre os termos hedônicos "gostei ligeiramente" (6) e "gostei moderadamente" (7). Com informação, o escore médio $(5,6)$ variou entre os termos "indiferente" (5) e "gostei ligeiramente" (6). Tais observações se aproximam das obtidas por Zandstra et al. (2001) no grupo de indivíduos com baixo interesse pela saúde, cujo escore hedônico não se modificou em função da informação light. Porém, no presente trabalho, observou-se interferência levemente negativa na resposta hedônica dos consumidores $(p<0,05)$, não tendo sido observado modificação significativa na intenção de compra desses indivíduos $(p>0,05)$. Tal fato pode estar relacionado com o maior interesse desses consumidores pelo aspecto sensorial do que pelo aspecto nutricional do alimento, visto que eram consumidores jovens, gozando de boa saúde e sem restrição alimentar.

\section{Conclusão}

O estudo comparativo dos perfis sensoriais das salsichas tipo Frankfurt comercializadas no mercado brasileiro indicou que os produtos tradicionais apresentaram características de sabor e aroma mais acentuados que as produzidas com carne de aves. A informação nutricional quanto ao baixo conteúdo de gordura ou light interferiu negativamente no status afetivo das salsichas produzidas com carne de aves, não interferindo na atitude de freqüência de uso, em consumidores com idade variando entre 20 e 25 anos. 


\section{Referências}

BARBUT, S.; MITTAL, G.S. Effects of three cellulose gums on the texture profile and sensory properties of low-fat frankfurters. International Journal of Food Science and Technology, v. 31, n. 4, p. 241-247, 1996.

CHAVES, J.B.P.; SPROESSER, R.L. Práticas de Laboratório de Análise Sensorial de Alimentos e Bebidas. Imprensa Universitária. UFV. Viçosa, MG, 1996, $81 \mathrm{p}$.

DAMÁSIO, M.H.; COSTELL, E. Análisis sensorial descriptivo: Generación de descriptores y selección de catadores. Rev. Agroquim. Tecnol Aliment., v. 31, n. 2, p. 165-178, 1991.

HUERTA-LEIDENZ, N.; JEREZ-TIMAURE, N.; MORÓN-FUENMAYOR, O.; URDANETA, E.R.; CARO, R. Experiencias en el entrenamiento de un panel de degustación de carne vacuna a nivel de un matadero frigorífico industrial venezolano. Archivos Latinoamericanos de Nutrición, v. 46, n. 1, p. 47-53, 1996.

KRYSTALLIS, A.; ARVANITOYANNIS, I.S.; KAPIRTI, A. Investigating Greek consumers' attitudes towards low-fat food products: a segmentation study. International Journal of Food Sciences and Nutrition, v. 54, n. 3, p. 219-233, 2003.
MARQUEZ, E.J.;AHMED, I.M.; WEST, R.L.; JOHNSON, D.D. Emulsion stability and sensory quality of beef frankfurters produced at different fat or peanut levels. Journal of Food Science, n. 54, p. 867-870, 1989. MOSKOWITZ, H.R. Product testing and sensory evaluation of foods. Marketing and R \& D approaches. Food and Nutricion Press Inc., Westort, Conn. 1983.605 p.

ORDÓÑEZ, M.; ROVIRA, J.; JAIME, I. The relationship between the composition and texture of conventional and low-fat frankfurters. International Journal of Food Science and Technology, v. 36, n. 7, p. 749-758, 2001.

SAS Institute. SAS User's Guide. 6.04 Edition. SAS Institute Inc., Cary, NC.1999.

SOLHEIM, R.; LAWLESS, H.T. Consumer purchase probability affected by attitude towards low-fat foods, liking, private body conciouness and information on fat and price. Food Quality and Preference, v. 7, n. 2, p. 137-143, 1996.

STONE, H.; SIDEL, J.L. Sensory evaluation praticez. 2. ed. Academic Press, 1993. $337 \mathrm{p}$.

ZANDSTRA, E.H.; GRAAF, C. de; VAN STAVEREN, W.A. Influence of health and taste attitudes on consuption of low and high fat food. Food Quality and Preference, v. 12, p. 75-82, 2001. 